ORIGINAL ARTICLE

\title{
UK population based study of severe retinopathy of prematurity: screening, treatment, and outcome
}

\author{
L Haines, A R Fielder, H Baker, A R Wilkinson
}

Arch Dis Child Fetal Neonatal Ed 2005;90:F240-F244. doi: 10.1136/adc.2004.057570

See end of article for authors' affiliations

.....................

Correspondence to: Professor Wilkinson, Department of Paediatrics, John Radcliffe Hospital, University of Oxford, Oxford OX3 9DU, ÚK; andrew.wilkinson@ paediatrics.ox.ac.uk

Accepted

22 November 2004

\begin{abstract}
Background: Retinopathy of prematurity (ROP) is one of the few causes of childhood blindness in which severe vision impairment is largely preventable. Ophthalmic screening for ROP is required to identify disease that requires treatment whereby the development of potentially blinding disease can be minimised. Objectives: To make the first UK population based estimate of the incidence of babies with severe ROP (stage 3 or more); to document their clinical characteristics and management and to evaluate the appropriateness of current ROP screening guidelines in the UK.

Patients: Cases were recruited through a national surveillance programme with 1 year ophthalmic follow up and data from clinician completed questionnaires.

Results: Between 1 December 1997 and 31 March 1999, 233 preterm babies with stage 3 ROP were identified. Severity (location, extent, and presence of plus disease) was associated with degree of prematurity, most severe in the most premature babies. Fiffy nine percent were treated. The UK screening protocol was followed in two thirds of cases, but in the remainder it was begun too late or was too infrequent. Three quarters of the cases were followed up at 1 year, and $13 \%$ had a severe vision deficit as a result of ROP.

Conclusions: Visual deficit as a result of ROP in premature babies continues to be a severe disability in some of the survivors of neonatal intensive care. Further efforts are needed to organise treatment regionally to improve outcome and standards of practice.
\end{abstract}

R etinopathy of prematurity (ROP), a condition confined to the developing retinal vascular system of preterm babies, is one of the few causes of childhood blindness in which severe vision impairment is largely preventable. Babies at risk of ROP require ophthalmic screening to identify disease requiring treatment, and this can prevent, although not entirely eliminate, the development of potentially blinding disease.

ROP is described by severity (stages $1-5$ ), location by zone (I-III), extent by sector, and by the presence of "plus" disease (fig 1). ${ }^{1}$ Severity stages 1 and 2 are mild because, unless they progress to stage 3, they resolve spontaneously without disabling sequelae. ${ }^{2}$ Stages $3-5$ are severe, as stage 3 is the first with significant risk of poor visual outcome, and stages 4 and 5 (being associated with retinal detachment) have a dismal prognosis for vision. A subdivision of stage 3, "threshold" ROP (fig 1), has a risk of blindness of about $50 \%$ if untreated and was the indication for treatment by laser or cryotherapy ${ }^{3}$ until late 2003 when treatment at an earlier stage was recommended. ${ }^{4}$

The International Classification of $\mathrm{ROP}^{15}$ stimulated considerable research, and the CRYO-ROP multicenter study in 1988 provided the first firm evidence that treatment was effective in reducing blindness. ${ }^{3}$ UK guidelines for screening and treatment of babies with birth weight $<1500 \mathrm{~g}$ and/or $<31$ weeks gestational age were published. ${ }^{67}$ In England and Wales in 2001, there were about 7500 live births under $1500 \mathrm{~g}^{8}$ who needed screening for ROP, many on several occasions. However, although most extremely preterm babies develop some degree of ROP, severe disease is relatively rare. In a multicentre study, $66 \%$ of babies under $1251 \mathrm{~g}$ developed ROP, but only $18 \%$ reached stage 3 , and $6 \%$ required treatment. ${ }^{3}$ Although ROP screening is a considerable workload for ophthalmologists and neonatal teams, most ophthalmologists rarely see severe disease.
A five year, three phase programme of research and education was funded by the Department of Health of England to improve identification and treatment of ROP in the UK. Phase one was a UK wide survey of services for ROP screening and treatment to audit adherence to national guidelines. ${ }^{910}$ Phase two comprised a series of multiprofessional educational workshops. Phase three was a multicentre UK study of severe ROP, which is reported here. Darlow ${ }^{11}$ reported a national study of ROP in New Zealand, but this is the first UK national study since ROP treatment started, and was undertaken to establish the incidence of stage 3 ROP, the characteristics of the population developing severe disease, the effects on vision at 1 year, and the impact of implementation of the UK screening guidelines.

\section{METHODS}

Cases of ROP at stage 3 or worse diagnosed between 1 December 1997 and 31 March 1999 were reported through various channels. The British Ophthalmological Surveillance Unit of the Royal College of Ophthalmologists actively sought reports from all consultant or associate specialist ophthalmologists in the UK. To identify cases known to neonatologists but not reported by ophthalmologists, a retrospective six monthly survey of all UK neonatal units was carried out. Other reports were obtained directly from ophthalmologists and coordinators of local surveys. Inevitably the total represents an underestimate of the total number of cases. Figure 2 shows the outcome of all babies reported during the study.

Parental consent was sought from the parents of all surviving babies. Neonatologists completed questionnaires about the babies' clinical condition, and ophthalmologists provided details of all screening examinations and any treatment. Ophthalmologists were also asked to perform a vision assessment at 1 year of age, corrected for gestational age, and to record the results in a second questionnaire that 


\section{Severity by stage ( 1 to 5 )}

Acute ROP develops at the growing tips of the developing blood vessels

STAGES 1 AND 2 (MILD ROP). Demarcation line and ridge respectively are seen at the junction of vascularised and avascular retina. Mild ROP resolves spontaneously without significant sequelae

STAGES 3-5 (SEVERE ROP). Stage 3: ridge with extraretinal fibrovascular proliferation, and carries a significant risk of adverse visual outcome. Stages 4 and 5 represent partial and total retinal detachment respectively and result in severe permanent visual impairment.

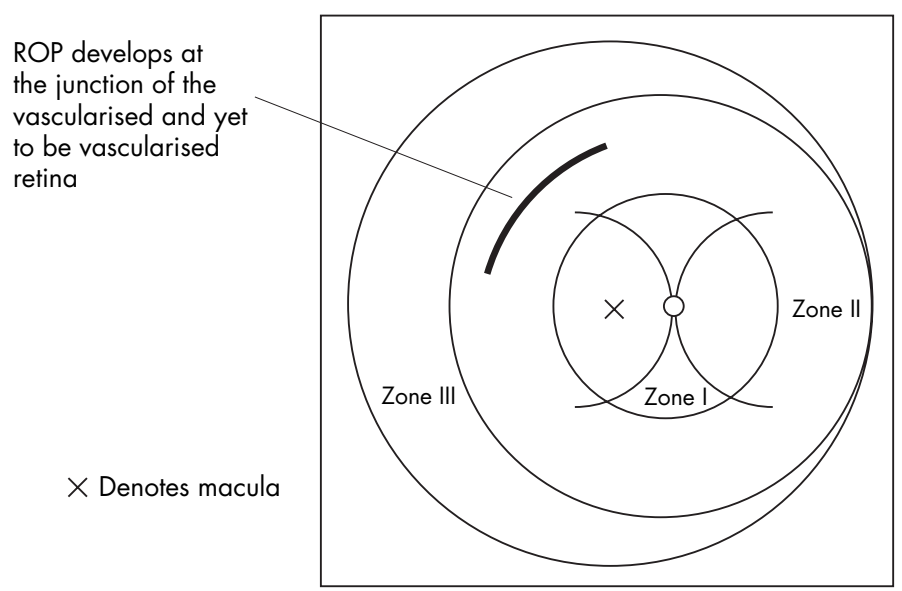

\section{Location by zone}

Retinal blood vessels grow out from the optic disc in zone I towards the periphery (zone III), thus the zone vascularised reflects maturity. ROP in zone I affects the most immature baby and is very likely to become severe with a poor outcome, whereas ROP located in zone III carries a very low risk for severity and adverse outcome

\section{Extent}

ROP extent around the retinal circumference is recorded in "clock hours" 1-12

\section{4. "Plus" Disease}

ROP activity is reflected by engorgement and tortuosity of the retinal and iris blood vessels. These are powerful indications that ROP is, or will become, severe

\section{"Threshold" ROP}

At least 5 continuous or 8 cumulative clock hours of stage 3 ROP in zones I or II, in the presence of "plus" disease. Threshold ROP was the indication for treatment by cryotherapy or laser during the period of this study, but see Rahi and Dezateux ${ }^{25}$ for recently revised recommendations

sought information on refractive state, structural ophthalmic abnormalities, and vision. Tests of the latter were necessarily qualitative (perception of light, ability to fix and follow) and were supplemented, where possible, by results of preferential looking-based tests.

Ethical approval was obtained from the South Thames Multicentre Research Ethics Committee and from local research ethics committees.

\section{RESULTS}

Reports were received of 401 potential cases of stage 3 ROP. After duplicates or invalid reports (87) and those where ROP status could not be ascertained (62) were excluded, there were 252 confirmed cases of stage 3 or worse ROP in 15 months reported by 129 individual ophthalmologists. Insufficient information (16) and refusal of consent (2) reduced the number to 234 (fig 2 ).
Figure 1 Descriptors of retinopathy of prematurity (ROP). 


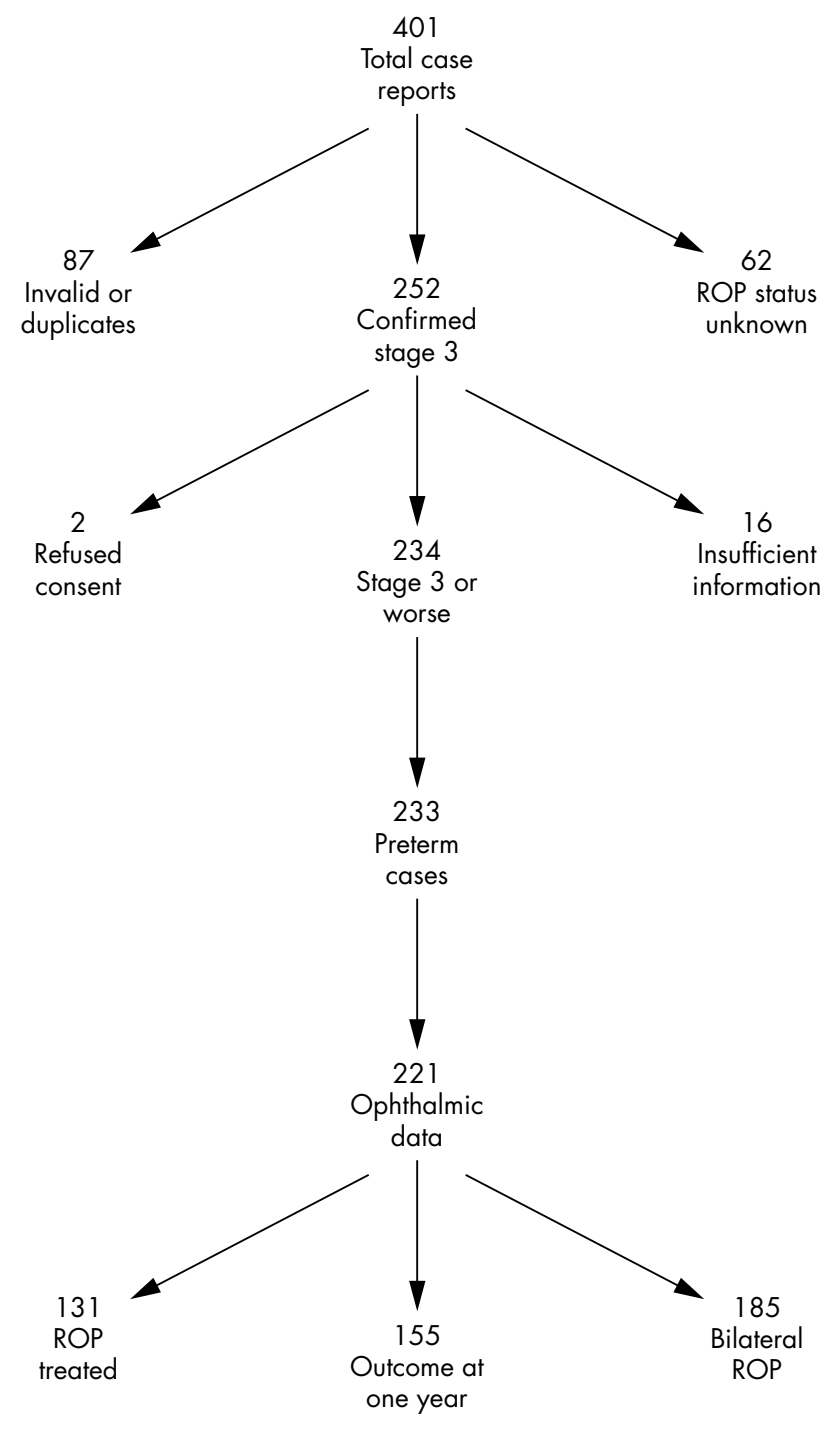

Figure 2 Outcome of all babies reported to have retinopathy of prematurity (ROP) stage 3 or worse from 1 December 1997 to 31 March 1999.

screening examination, and five babies already had stage 4 or 5 by the time of presentation.

In Table 1, the maximum severity of ROP in each stage 3 eye is categorised by the most central zone involved, maximum circumferential extent of ROP, and the presence of plus disease. The results are shown for 382 eyes which could be categorised with the mean birth weight and gestational age for babies in each group (using the more severe eye in babies with asymmetric disease) and the proportion treated. Disease affecting zone II was most common, $85 \%$ of eyes (324/382). In $11 \%(42)$, stage 3 ROP was restricted to zone III, and only $4 \%$ (16) had zone I disease, which is the most posterior zone and associated with poorest outcome. Babies with lowest gestational age and birth weight had the more severe ROP (table 1).

\section{ROP treatment}

Fifty nine percent of the cohort (131/221) were treated; treatment was bilateral in $121(92 \%)$ and unilateral in 10 $(8 \%)$. Eighteen babies required more than one treatment (16 twice and two three times). The decision to treat was made at a mean postnatal age of 11.7 (2.3) weeks (median 11.7; range
0.4-18.1) and a mean postmenstrual age of 37.4 weeks (range 32.6-50).

The reason for intervention was assessed for 237/250 treated eyes. For $181(76 \%)$, the indication was "threshold ROP" (fig 1); 56 (24\%) eyes were treated below threshold, and for $16 / 127$ babies (13\%) neither eye was at threshold when treated. The reasons for this are not fully known, but two had zone I ROP and two were treated below threshold to avoid the baby having a second general anaesthetic. In 18 babies with asymmetric disease, treatment of the less affected eye was brought forward.

Thirty nine ophthalmologists treated the 131 babies, five treated nine or more, and 10 treated three to six, while 24 ophthalmologists treated only one or two babies. Where treatment technique was known (123), 76\% were treated by laser, $22 \%$ by cryotherapy, and $2 \%$ by both.

\section{UK guidelines screening and treatment protocol}

The current UK guidelines specify a screening criteria of $\leqslant 1500$ g birth weight and/or $\leqslant 31$ weeks gestational age. ${ }^{6}$ No babies in this study fell outside both these criteria, although two were outside the birth weight criteria (1806 g and $2300 \mathrm{~g}$ ) and one outside the gestational age criteria (33 weeks 6 days).

The screening protocol specifies a first examination at 6-7 weeks postnatal age and then at least every two weeks until vascularisation has progressed into zone III. ${ }^{7}$ Records of screening history for 193 cases were assessed for adherence to the protocol. The protocol had been followed in 72\% (139/ $193)$; in $28 \%$ deviations from the protocol occurred where either screening was initiated too late (29) or was too infrequent (25). The assessment also identified 13 babies in whom the progression of ROP indicated that an inaccurate recording of ROP severity had been made. I year vision assessments were available for $72 \%(39 / 54)$ of cases where screening protocol had not been followed, and in three cases non-adherence was considered to be the cause of visual morbidity at 1 year.

Guideline treatment criteria specify that, when treatment is indicated, it should ideally be undertaken within three days. The mean (SD) interval between the decision to treat and treatment in this study was 1.5 (1.5) days; in five babies

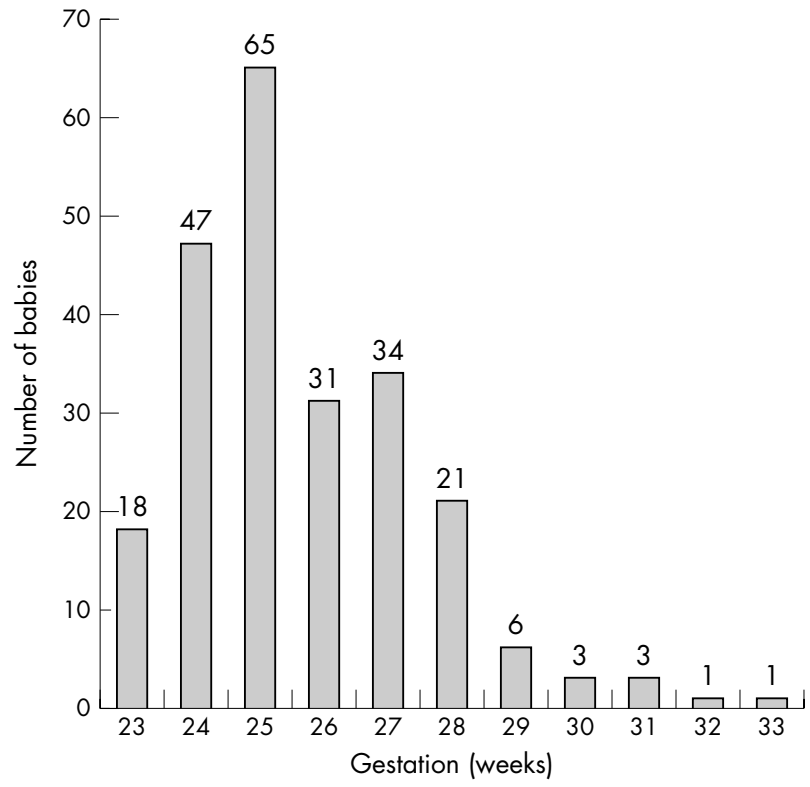

Figure 3 Number of cases of retinopathy of prematurity stage 3 or worse by gestational age. 


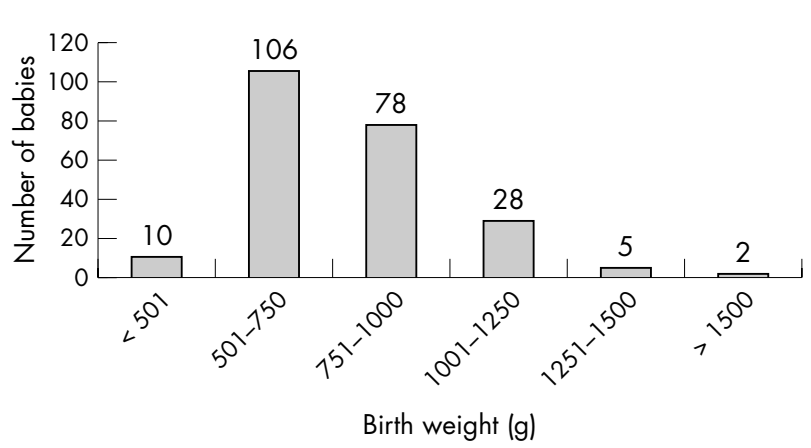

Figure 4 Distribution of cases of retinopathy of prematurity stage 3 or worse by birth weight.

the ophthalmologist reported suboptimal intervals (2-6 days) because of poor clinical condition (2) or lack of anaesthetic support (1), intensive care facilities (1), or ophthalmic cover ( 1 ).

\section{Outcome at 1 year}

Seventeen infants died before their first birthday. Of the remaining 216 infants, $75 \%$ were followed up. Although comparable to other studies of preterm babies, the possibility of bias has to be considered. Other studies have found a higher prevalence of visual abnormalities (strabismus and or cicatricial ROP) in those whose families were reluctant to attend for follow up.

Table 2 shows a comparison of the follow up group with the group lost to follow up (excluding those who died). Those followed up had a significantly greater gestational age $(\mathrm{p}<0.05)$, a slightly, although not significantly, higher birth weight, and were more likely to have been treated than those lost to follow up.

Assessments at 1 year were completed for $72 \%$ (155/216). Mean age at follow up was 1 year 5 months; 59\% (92/155) had been treated for ROP.

Nineteen percent (29) had some degree of vision loss. Six were bilaterally and 11 unilaterally blind, and 12 had reduced vision in one (7) or both eyes (5). There was enough information for 27 of the 29 cases to establish cause of vision loss, and in $74 \%(20)$ this was a consequence of ROP rather than of neurological insult. ROP induced vision deficit included four with bilateral blindness and 10 with unilateral blindness. Twenty four (83\%) with vision loss had been treated for ROP.

The initial ophthalmic records of the surviving 61 babies lost to follow up identified three cases (two with retinal detachment, one unilateral, and one with bilateral retinal folds) who would have been blinded by ROP.

\section{DISCUSSION}

This multicentre study provides an overview of UK screening and treatment practice for ROP during the period 1997-1999.
Table 2 Comparison of subjects followed up with those lost to follow up

\begin{tabular}{lll}
\hline Characteristic & Followed up & Not followed up \\
\hline Sex ratio (M:F) & $53 \%(80 / 152)$ & $54 \%(29 / 54)$ \\
Median GA (weeks) & 25.9 & 25.1 \\
Mean birth weight (g) & 770 & 710 \\
$\%$ treated & $59.7(89 / 149)$ & $54.4(31 / 57)$ \\
\hline GA, Gestational age. & & \\
\hline
\end{tabular}

The 233 cases recruited constitute one of the largest studies of severe ROP reported and gives a national population based perspective on the characteristics of babies developing stage 3 ROP and their ophthalmic outcome at 1 year.

Although the study data were provided from routine clinical notes and could not be independently validated, the study findings correlate well with other studies using trained study observers particularly in relation to birth weight and gestational age of babies at risk, the association of severity with prematurity, the incidence of zone I disease, and, using treatment as a proxy, the timing of development of threshold disease.

Some under-ascertainment is likely. At the time of the study, only $65-70 \%$ of consultant ophthalmologists were participating in the newly established British Ophthalmic Surveillance Unit reporting scheme, ${ }^{13}$ and the research team were aware that non-responders included a very small number of ROP active clinicians. However, a subsequent evaluation of the completeness of case ascertainment for studies using the British Ophthalmic Surveillance Unit estimated that $91 \%$ of cases of ROP had been reported. ${ }^{13}$

The study findings also inform the debate about criteria for screening, mindful that these should be periodically reviewed in the light of new evidence. The current UK guidelines recommend examination of all babies born at $<31$ weeks and/or $<1500 \mathrm{~g}$, which would capture virtually all babies at risk of severe ROP in the $\mathrm{UK}^{14-17}$ and other developed countries, such as Canada, ${ }^{18}$ USA, ${ }^{19}$ and Sweden. ${ }^{20}$ Table 2 shows that the use of birth weight and gestational age together and not singly brings in outliers and offers the possibility of reducing screening criteria to $<29$ weeks gestational age and/or $<1250 \mathrm{~g}$, although this would have excluded four babies in the current study, one requiring treatment. However, occasionally babies well outside any birth weight and gestational age criteria develop ROP requiring treatment, ${ }^{18}$ and currently there seems to be no method of identifying these babies by other criteria. Comparison of populations developing threshold disease has also shown that screening criteria applicable to high human development communities may not be appropriate in low human development countries where babies with a wider range of birth weight and gestational age are at risk of developing severe ROP. ${ }^{21}$

\begin{tabular}{lllll} 
Table 1 & Stage 3 retinopathy of prematurity: zone, severity, extent, and treatment \\
\hline & $\begin{array}{l}\text { Eyes } \\
\text { (n= 382) }\end{array}$ & $\begin{array}{l}\text { Birth weight } \\
\text { (g) }\end{array}$ & $\begin{array}{l}\text { GA } \\
\text { (weeks) }\end{array}$ & $\begin{array}{l}\text { Proportion of eyes } \\
\text { treated }\end{array}$ \\
Stage 3 severity & $16(4 \%)$ & $671(130)$ & 24.5 & $88 \%(14 / 16)$ \\
\hline Zone I & $71(18 \%)$ & $740(148)$ & 25.3 & $96 \%(189 / 197)$ \\
Zone II: 9-12 hours (plus disease) & $126(33 \%)$ & $784(283)$ & 25.5 & \\
Zone II: 5-8 hours (plus disease) & $48(13 \%)$ & $789(158)$ & 26.0 & $19 \%(24 / 127)$ \\
Zone II: $1-4$ hours (plus disease) & $79(21 \%)$ & $854(208)$ & 26.3 & $19 \%(8 / 42)$ \\
Zone II: no plus disease & $42(11 \%)$ & $871(304)$ & 26.4 & $19 \%(11)$ \\
Zone III & & & &
\end{tabular}

Values for birth weight are mean (SD).

GA, Gestational age. 
A number of study babies had severe ROP diagnosed at the first examination, one at 4 weeks and 11 at 6-7 weeks postnatal age. ROP onset and progression are both determined largely by postmenstrual age rather than neonatal events, ${ }^{22}{ }^{23}$ a fact not adequately recognised in the current guidelines, which recommend that screening for all starts at 6-7 postnatal weeks. ${ }^{7}$ Reynolds et $a l^{24}$ proposed that guidelines should recommend earlier examinations in more mature babies, which would have introduced a margin of safety for the babies referred to above.

This study also documents a change in services since $1994 .^{8}$ Fewer ophthalmologists are treating ROP (39v65), and laser therapy has become the treatment of choice. Although it is encouraging that fewer ophthalmologists are treating ROP, in line with the recommendations that treatment services should cover larger geographical populations, ${ }^{9}{ }^{17}$ almost two thirds of ophthalmologists treating ROP in 1998/9 treated two or less babies. Further moves should be made to reorganise treatment services to ensure that surgeons develop sufficient expertise, particularly in view of the recent recommendation that treatment is performed at an earlier stage, ${ }^{4}$ which will increase the number of babies requiring treatment.

Treatment for ROP was shown to be effective in 1988, and this generated a wave of activity including the production of UK guidelines for ROP screening. ${ }^{6}$ In 1995, the guidelines were revised ${ }^{7}$ to include ROP treatment, and a number of multiprofessional educational workshops were held between 1995 and 1997. A national audit in 1995 found that ROP screening was taking place in $96 \%$ of UK neonatal units. ${ }^{9}$ The UK is probably the only country in which causes of childhood vision impairment have been evaluated on a number of occasions, both before and after the introduction of ROP treatment. Between 1969 and 1976 and 1976 and 1985, the incidence of ROP induced severe vision impairment as a proportion of childhood vision impairment was stable at 5\%, but rose to $8 \%$ between 1985 and $1990 .{ }^{25}$ The incidence then decreased to $3 \%$ in $2000,{ }^{26}$ when 13 UK cases were reported, a figure very similar to the number of babies with severe visual impairment reported in this study. Although these studies are not directly comparable, if treatment were not effective, with the increased survival of extremely preterm babies, ${ }^{27}{ }^{28}$ the incidence of ROP induced vision impairment would have been expected to increase rather than decrease.

Although extremely preterm babies are at risk of a range of adverse neurodevelopmental disorders, ${ }^{27}$ a systematic protocol of screening and treatment can reduce the potential burden of disability from ROP. This study shows that, in the UK, most babies are screened according to the protocol, and only in very few cases did non-adherence to the protocol result in visual morbidity. Because certain visual deficits do not become apparent until an older age, assessment at 1 year underestimates the total long term visual deficit. ${ }^{4}$ However, at least 17 study babies were blind in one or both eyes as a result of ROP, indicating that there is still substantial lifelong visual disability in the UK resulting from ROP. Close collaboration between ophthalmologists, neonatologists, and nursing staff in neonatal intensive care units will ensure that all babies at risk benefit from this effective preventive screening programme.

\section{ACKNOWLEDGEMENTS}

We thank all clinicians and the British Ophthalmological Surveillance Unit for helping us to recruit patients and for completing the study questionnaires. We also thank the parents for consent to include their baby in this study.

\section{Authors' affiliations}

L Haines, H Baker, Royal College of Paediatrics and Child Health, London, UK

A Fielder, Department of Ophthalmology, Imperial College London, UK A R Wilkinson, Department of Paediatrics, John Radcliffe Hospital, University of Oxford, Oxford, UK

The study was funded by the Department of Health of England who did not play any part in the design, collection, analysis, and interpretation of the data.

Competing interests: none declared

\section{REFERENCES}

1 Committee for the Classification of Retinopathy of Prematurity. An international classification of retinopathy of prematurity. Br J Ophthalmol 1984;68:690-7.

2 O'Connor AR, Stephenson T, Johnson A, et al. Long term ophthalmic outcome of low birth weight children with and without retinopathy of prematurity. Pediatrics 2002;109:12-18.

3 Cryotherapy for Retinopathy of Prematurity Cooperative Group. Multicenter trial of cryotherapy for retinopathy of prematurity: preliminary results. Arch Ophthalmol 1988;106:471-9.

4 Early Treatment for Retinopathy of Prematurity Cooperative Group. Revised indications for the treatment of retinopathy of prematurity. Arch Ophthalmol 2003;121:1684-96

5 The International Committee for the Classification of the Late Stages of Retinopathy of Prematurity. An international classification of retinopathy of prematurity. II. The classification of retinal detachment; Arch Ophthalmol 1987; 105:906-12.

6 Anon. College News: ROP screening duty. Quarterly Bulletin of the College of Ophthalmology 1990;(autumn):6.

7 The Royal College of Ophthalmologists, British Association of Perinatal Medicine. Retinopathy of prematurity: guidelines for screening and treatment. Early Hum Dev 1996;46:239-58.

8 Office of National Statistics. 2001. www.statistics.gov.uk (accessed 22 Feb 2005).

9 Haines L, Fielder AR, Pollock Jl, et al. Retinopathy of prematurity in the UK: the organisation of services for screening and treatment. Eye 2002;16:33-8.

10 Fielder AR, Haines L, Scrivener R, et al. Retinopathy of prematurity in the UK. II. Audit of national guidelines for screening and treatment. Eye 2002; 16:285-91.

11 Darlow BA. Incidence of retinopathy of prematurity in New Zealand. Arch Dis Child 1988;63:1083-6.

12 Larsson E, Carle-Petrelius B, Cernerud G, et al. Incidence of ROP in two consecutive Swedish population based studies. Br J Ophthalmol 2002;86: 1122-6

13 Foot B, Stanford M, Rahi J, et al. The British Ophthalmological Surveillance Unit: an evaluation of the first 3 years. Eye 2003;17:9-15.

14 Fleck BW, Wright E, Dhillon B, et al. An audit of the 1995 Royal College of Ophthalmologists guidelines for screening of retinopathy of prematurity applied retrospectively in one regional neonatal intensive care unit. Eye 1995:9:31-5.

15 Goble RR, Jones HS, Fielder AR. Are we screening too many babies for retinopathy of prematurity? Eye 1997;11:509-14.

16 Mathew MRK, Fern Al, Hill R. Retinopathy of prematurity: are we screening too many babies? Br J Ophthalmol 2002;16:538-42.

17 Brennan R, Gnanaraj L, Cottrell DG. Retinopathy of prematurity in practice. 1. Screening for threshold disease. Eye 2003;17:183-8.

18 Lee SK, Normand C, McMillan D, et al. Evidence for changing guidelines for routine screening for retinopathy of prematurity. Arch Pediatr Adolesc Med 2001;155:387-95.

19 Wright K, Anderson ME, Walker E, et al. Should fewer premature infants be screened for retinopathy of prematurity in the managed care era? Pediatrics 1998;102:31-4.

20 Andruscavage L, Weissgold DJ. Screening for retinopathy of prematurity. Br J Ophthalmol 2002;86:1127-30.

21 Gilbert C, Rahi J, Eckstein M, et al. Retinopathy of prematurity in middleincome countries. Lancet 1997;350:12-14.

22 Palmer EA, Flynn JT, Hardy RJ, et al. The Cryotherapy for Retinopathy of Prematurity Cooperative Group. Incidence and early course of retinopathy of prematurity. Ophthalmology 1991;98:1628-40.

23 Fielder AR, Shaw DE, Robinson J, et al. Natural history of retinopathy of prematurity: a prospective study. Eye 1992;6:233-42.

24 Reynolds JD, Dobson V, Quinn GE, et al. Evidence-based screening for retinopathy of prematurity: natural history data from CRYO-ROP and LIGHTROP Studies. Arch Ophthalmol 2002;120:1470-6.

25 Rahi J, Dezateux C. Epidemiology of visual impairment. Arch Dis Child 1998;78:381-6.

26 Rahi J, Cable N. Lancet 2003;362:1359-65.

27 Wood NS, Marlow N, Costeloe K, et al. Neurologic and developmental disability after extremely preterm birth. N Engl J Med 2000;343:378-84

28 CESDI. Project 27/28: an enquiry into quality of care and its effect on the survival of babies born at 27-28 weeks. www.cemach.org.uk (accessed 22 Feb 2005). 